\title{
BMJ Open Impact of massive blood transfusion during adult extracorporeal membrane oxygenation support on long-term outcomes: a nationwide cohort study in Taiwan
}

Fang-Ting Chen (10 , ${ }^{1}$ Shao-Wei Chen, ${ }^{2,3}$ Victor Chien-Chia Wu, Kuo-Chun Hung, ${ }^{2}$ Shang-Hung Chang, ${ }^{2,3}$ Pei-Chi Ting, ${ }^{1}$ An-Hsun Chou (i) ${ }^{1,3,5}$

To cite: Chen F-T, Chen S-W, Wu VC-C, et al. Impact of massive blood transfusion during adult extracorporeal membrane oxygenation support on long-term outcomes: a nationwide cohort study in Taiwan. BMJ Open 2020;10:e035486. doi:10.1136/ bmjopen-2019-035486

- Prepublication history and additional material for this paper are available online. To view these files, please visit the journal online (http://dx.doi. org/10.1136/bmjopen-2019035486).

F-TC and S-WC contributed equally.

Received 03 November 2019 Revised 27 March 2020 Accepted 11 May 2020
Check for updates

(C) Author(s) (or their employer(s)) 2020. Re-use permitted under CC BY-NC. No commercial re-use. See rights and permissions. Published by BMJ.

For numbered affiliations see end of article.

Correspondence to Dr An-Hsun Chou; f5455@cgmh.org.tw

\section{ABSTRACT}

Objectives Bleeding is a common problem during adult extracorporeal membranes oxygenation (ECM0) support, requiring blood transfusion for correction of volume depletion and coagulopathy. The goal of this study is to investigate the long-term outcomes for adults under support of ECMO with massive blood transfusion (MBT). Design Retrospective nationwide cohort study.

Setting Data were provided from Taiwan National Health Insurance Research Database (NHIRD).

Participants and interventions Totally 2757 adult patients were identified to receive MBT (red blood cell $\geq 10$ units) during ECM0 support from 2000 to 2013 via Taiwan NHIRD.

Main outcome measures The outcomes included inhospital major complications/mortality, all-cause mortality, cardiovascular death, newly onset end-stage renal disease and respiratory failure during the follow-up period. Results Patients with MBT had higher in-hospital mortality $(65.6 \%$ vs $52.1 \%$; OR $1.74 ; 95 \% \mathrm{Cl} 1.53$ to 1.98$)$ and all-cause mortality during the follow-up (47.0\% vs $35.8 \%$; HR 1.46; $95 \% \mathrm{Cl} 1.25$ to 1.71) than those without MBT. Not only higher incidences of post ECMO sepsis, respiratory failure and acute kidney injury, but also longer duration of ECMO support, ventilator use and intensive care unit stay were demonstrated in the MBT group. Moreover, a subdistribution hazard model presented higher cumulative of respiratory failure $(19.8 \%$ vs $16.2 \%$; subdistribution HR $1.36 ; 95 \% \mathrm{Cl} 1.07$ to 1.73 ) for the MBT cohort. Positive dose-dependent relationship was found between the amount of transfused red blood cell product and in-hospital mortality. In the MBT subgroup analysis for the impact of transfused ratio (fresh frozen plasma/ packed red blood cell) on in-hospital mortality, ratio $\geq 1.0$ had higher mortality.

Conclusions Patients with MBT during ECMO support had worse long-term outcomes than non-MBT population. The transfused amount of red blood cell had positive dosedependent effect on in-hospital mortality.

\section{INTRODUCTION}

Extracorporeal membrane oxygenation (ECMO) is a life-saving technique applied
Strengths and limitations of this study

- This study analyses the impact of massive blood transfusion (MBT) on extracorporeal membranes oxygenation (ECMO) patients during the long-term follow-up period.

- This study collected data using the National Health Insurance Research Database (NHIRD) derived from the government-operated singer-payer health insurance programme, covered nearly whole Taiwan residents.

- This article provides large-scale, observational results with control group comparison for multianalysis of short-term and long-term outcome among patients received MBT during ECMO support.

- The main limitations of this study are that NHIRD lacks detailed patient information, such physical status, laboratory data, image reports, intervention notes and ECMO parameters, which might affect the bleeding severity.

for mechanical circulatory and respiratory support for over four decades in the intensive care field. ${ }^{1}$ ECMO is regarded as a bridge to cardiopulmonary function recovery, transplantation or other mechanical support devices for patients with refractory cardiac failure, severe respiratory failure, extreme hypothermia, postcardiotomy shock, septic shock, toxic ingestion or trauma. ${ }^{23}$ Although ECMO is effective, high rates of associated complications and mortality remain to be debated. Serious complications include bleeding, neurological damage, infection and acute kidney injury, ${ }^{4}$ with bleeding statistically being the most common $(30 \%-60 \%){ }^{35}$

Because of anticoagulation therapy to prevent thromboembolic events and oxygenator thrombosis, patients under ECMO support are at risk for bleeding. ${ }^{6}$ Nevertheless, 
A

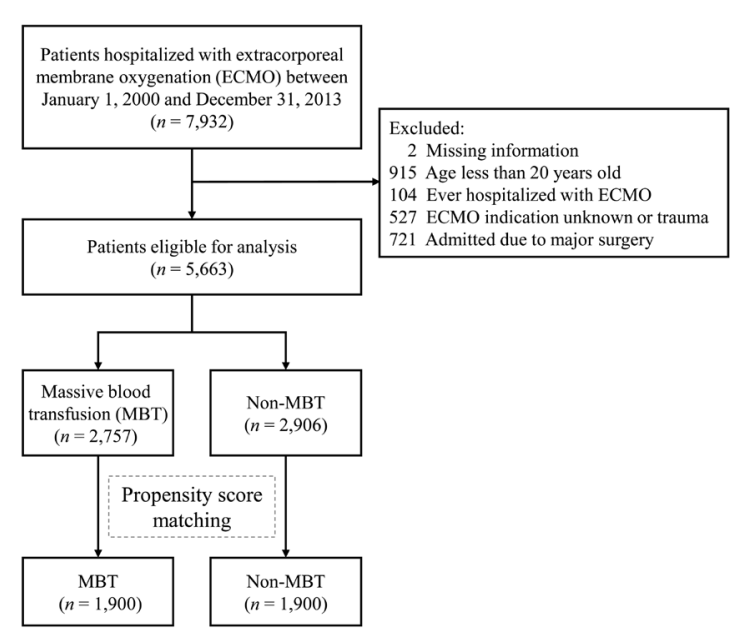

B

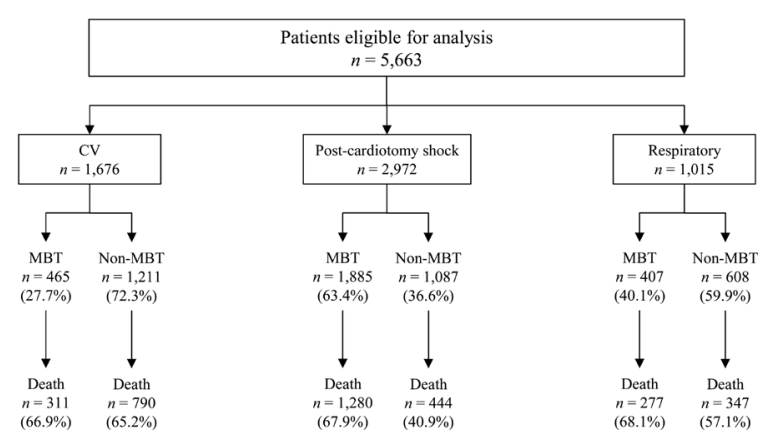

Figure 1 (A) The inclusion of the study patients. (B) The proportion of in-hospital mortality in each of the three main ECMO indications by the presence or absence MBT. CV, cardiovascular.

red blood cell (RBC) transfusions induce various adverse complications, such as profound coagulopathy, electrolyte/acid-base imbalance, hypothermia, transfusionrelated acute lung injury, infection and acute kidney injury. ${ }^{78}$ Massive blood transfusion (MBT) augments the negative outcomes. ${ }^{9}$

However, to our knowledge, no previous study has discussed the long-term outcomes of patients with MBT during ECMO support compared with a non-MBT group via a nationwide database. Therefore, this retrospective, population-based cohort study was designed to evaluate the in-hospital morbidity, mortality and long-term outcomes of adult ECMO patients receiving MBT.

\section{MATERIALS AND METHODS \\ Data source}

We analysed data from The National Health Insurance (NHI) Research Database (NHIRD), derived from the government-operated singer-payer NHI programme. The NHI programme was launched in 1995 and covered almost all Taiwan residents (99\%). In Taiwan, the costs of all lifesaving procedures, surgeries, medical treatments and medications were reimbursed in the NHIRD, such as use of ECMO, cardiac surgery and blood transfusion. ${ }^{10-13}$

\section{Study population}

This retrospective, population-based cohort study compared the outcomes between patients who did and did not receive MBT during ECMO support. MBT was defined as RBC transfusion of $\geq 10$ units within a 1 month period since the application of ECMO ${ }^{14}$ We reviewed the hospitalisation records of NHIRD from 1 January 2000 to 31 December 2013. Patients who received ECMO were identified via the NHIRD procedure code (68036) and International Classification of Disease, Ninth Revision, Clinical Modification (ICD-9-CM) procedure code (39.65).${ }^{15}$ Then, we excluded patients with incomplete data during the follow-up, age younger than 20 years, previous hospitalisation with ECMO experience, uncertain indication for ECMO, indication for ECMO due to trauma and admission for major surgery. Finally, a total of 5663 patients were eligible for further analysis (figure 1A).

\section{Comorbidities and outcome}

The demographic characteristics, indications and detailed information of ECMO use were identified by ICD-9-CM and NHI procedure codes for the index hospitalisation. A history of bleeding-related disease and other comorbidities were identified via the ICD-9-CM diagnostic codes for prior hospitalisation. A majority of the ICD-9-CM and NHI procedure codes were verified in previous studies. ${ }^{16}$ The outcomes of interest to this study included in-hospital complications and outcomes and time-to-event outcomes. Time-to-event outcomes were all-cause mortality, cardiovascular death (CV), end-stage renal disease (ESRD) or dialysis, and respiratory failure. All-cause mortality was defined by a withdrawal from the NHI. ${ }^{17}$ Dialysis and respiratory failure were verified by approval of possessing a catastrophic illness certificate card. ${ }^{18}$ The definition of CV death met the criteria of the Standardised Definitions for CV and Stroke Endpoint Events in Clinical Trials drafted by the United States Food and Drug Administration. All patients were followed from the index admission until 31 December 2013 or the date of death, whichever came first.

\section{Statistical analysis}

The MBT and non-MBT groups were compared by a propensity score matching (PSM). We matched each patient from the MBT group with a counterpart from the non-MBT group. The propensity score was the predicted probability to be in the MBT group, derived from a given multivariable logistic regression value of covariates. The covariates included in propensity score calculation were age, sex, ECMO indication, bleeding-related diseases or bleeding history (four items), comorbid conditions (12 items), hospital level, ECMO volume of the hospital and the index date of admission. The matching was processed using a greedy nearest neighbour algorithm with a calliper of 0.2 times the SD of the logit of propensity score and without replacement and with random matching order. We checked the quality of matching by using the absolute value of standardised mean difference (SMD) in which a value of 
less than 0.1 was considered to have negligible difference between the two groups.

The trend of MBT and in-hospital mortality rate across the study years (2000-2013) was assessed by Cochran-Armitage test. The in-hospital complications and outcomes between the two groups were compared using linear regression or logistic regression analyses, which considered the within-cluster correlation of the same matching pair by introducing the work correlation matrix of the generalised estimating equation (GEE). The study group was the only explanatory variable in the GEE model. After excluding patients who died during the index admission, we compared the risks of time-to-event outcomes between the two groups. The risk of all-cause mortality and the risk of other time-to-event outcomes (ie, respiratory failure) between the groups were compared using the Cox proportional hazard model and the Fine and Gray subdistribution hazard model, respectively; the latter model considered death as a competing risk. In the survival analyses, we additionally adjusted the propensity score to mitigate the confounders between the groups. ${ }^{19}$ The aforementioned PSM and analyses were further performed separately on different ECMO indications, which were classified into postcardiotomy cardiogenic shock (PCS) or non-PCS.

Finally, the dose-dependent relationship between the transfused ratio (fresh frozen plasma(FFP)/RBC), total packed RBC (PRBC) volume, and in-hospital mortality rate was assessed by a trend test of univariate logistic regression. A two-sided $\mathrm{p}<0.05$ indicates statistical significance, and multiple testing (multiplicity) was not adjusted. All statistical analyses, including the procedures of 'psmatch'for PSM, 'genmod' for GEE, and 'phreg' for survival analyses, were performed using the SAS V.9.4 (SAS Institute).

\section{Patient and public involvement}

No patient was involved in this study.

\section{RESULTS}

\section{Study population characteristics}

A total of 5663 patients who initially received ECMO between 1 January 2000 and 31 December 2013 (figure 1A) were categorised into the MBT $(n=2757$; average age, 59.6 years; males, $69.1 \%)$ and non-MBT $(\mathrm{n}=2906)$ groups. Table 1 presents the baseline characteristics of the study groups. The major indications for ECMO application in the MBT and non-MBT groups were PCS $(68.4 \%)$ and CV reasons $(41.7 \%)$, such as cardiogenic shock, myocarditis or acute myocardial infarction, respectively. The types of cardiac procedures for postcardiotomy were classified as follows: aortic surgery (15.8\%), valve surgery (14.9\%), coronary artery bypass graft $(33.0 \%)$, valve surgery combined with coronary artery bypass graft $(8.2 \%)$ and others $(28.1 \%)$. A significant difference was found in numerous variables between the two groups before PSM, with an absolute SMD value of $>0.1$ (table 1 , left panel).
However, the group difference was trivial after PSM (table 1, right panel).

Figure 1B displays the proportion of in-hospital mortality for the three main ECMO indications according to the presence or absence of MBT. Patients with MBT had higher in-hospital mortality rate than those without MBT. Figure 2 shows the epidemiology of ECMO application in Taiwan from 2000 to 2013. The volume of ECMO use increased gradually year by year $(\mathrm{p}<0.001)$. PCS remained the chief indication for ECMO support across the years. Notably, the proportion of MBT decreased annually $(p<0.001)$ without an increasing in-hospital mortality rate over time $(\mathrm{p}=0.513)$.

\section{In-hospital mortality and ECMO-related complications}

Table 2 displays the in-hospital outcomes of patients with and without MBT. Compared with the non-MBT group, the MBT group had a higher in-hospital mortality rate (65.6\% vs $52.1 \%$; OR $1.74 ; 95 \%$ CI 1.53 to 1.98 ) and a higher incidence of ECMO-related complications, including sepsis (OR 1.42; 95\% CI 1.21 to 1.67), respiratory failure (OR 1.27; 95\% CI 1.11 to 1.45 ) and acute kidney injury (OR 2.73; 95\% CI 2.37 to 3.13). Longer durations of ECMO support, ventilator use, intensive care unit (ICU) stay and hospitalisation stay, as well as a higher inpatient medical expenditure, were also noted in the MBT group than in the non-MBT group.

We further performed subgroup analysis according to ECMO indications, namely, non-PCS and PCS. No significant difference on the risk of in-hospital mortality was observed in patients with non-PCS indication (online supplementary table 1). In addition, the MBT group had greater risks of sepsis, respiratory failure and acute kidney than the non-MBT group. In patients with PCS indication, the MBT had higher in-hospital mortality risks (OR $2.81 ; 95 \%$ CI 2.31 to 3.41 ) and greater risks of sepsis and acute kidney injury than its counterpart (online supplementary table 2).

\section{Follow-up outcomes}

After excluding patients who died during the index admission, 653 and 911 patients remained in the MBT and non-MBT groups, respectively. During a median follow-up of 1.8 years (IQR 0.3-3.9 years), the MBT group exhibited a higher all-cause mortality rate than the nonMBT group (47.0\% vs $35.8 \%$, HR 1.46 ; $95 \%$ CI 1.25 to 1.71; figure $3 \mathrm{~A})$. In addition, the cumulative incidences of CVdeath and newly diagnosed ESRD were not significantly different between the two groups (figure 3B,C). Meanwhile, the cumulative incidence of respiratory failure was higher in the MBT group than in the nonMBT group (19.8\% vs $16.2 \%$; subdistribution HR 1.36; $95 \%$ CI 1.07 to 1.73 ; figure $3 \mathrm{D}$ )

Subgroup analysis according to ECMO indications, that is, non-PCS and PCS, was further performed. The risks of overall mortality after discharge, CV death and respiratory failure in patients with non-PCS indication had no significant difference (online supplementary 


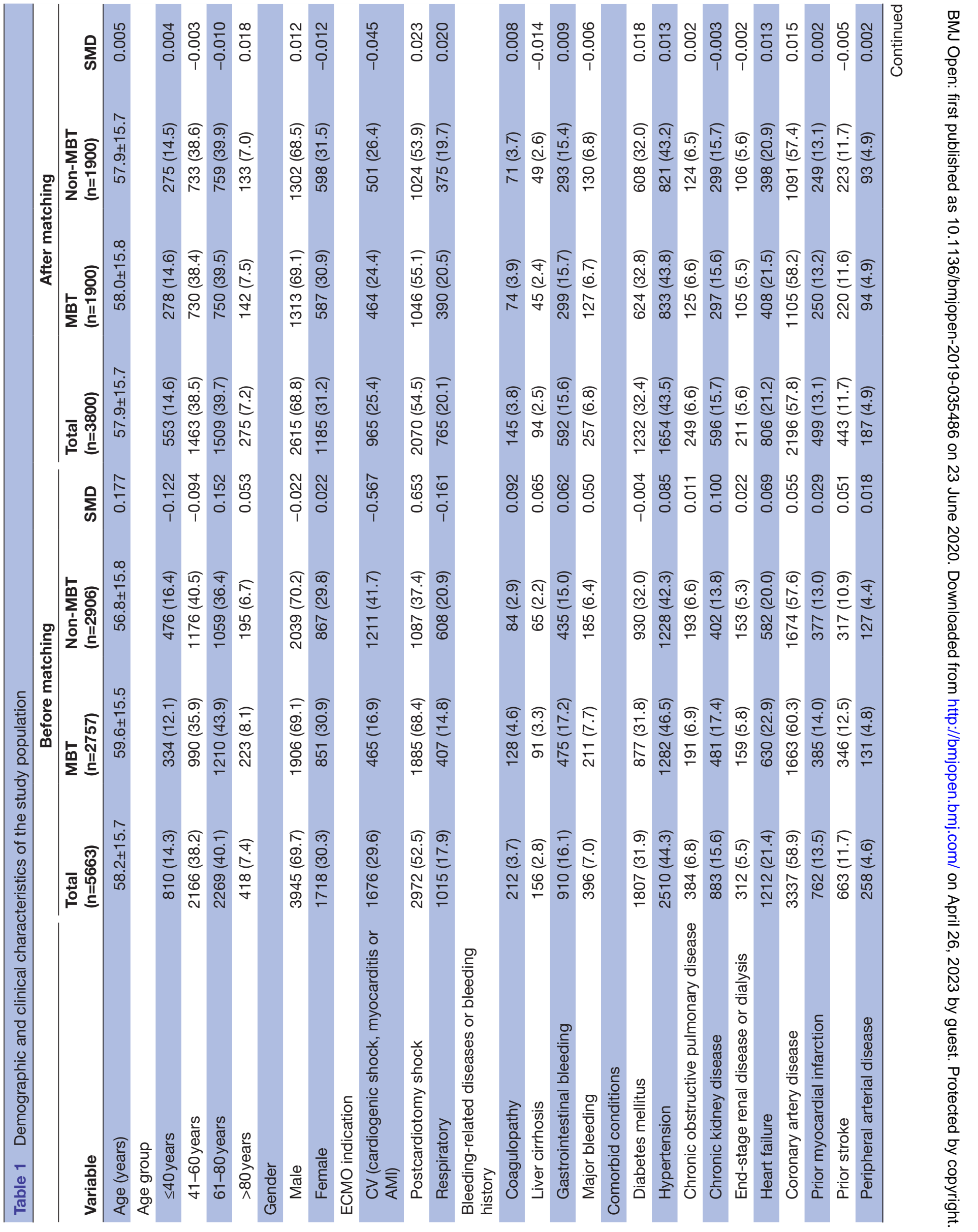




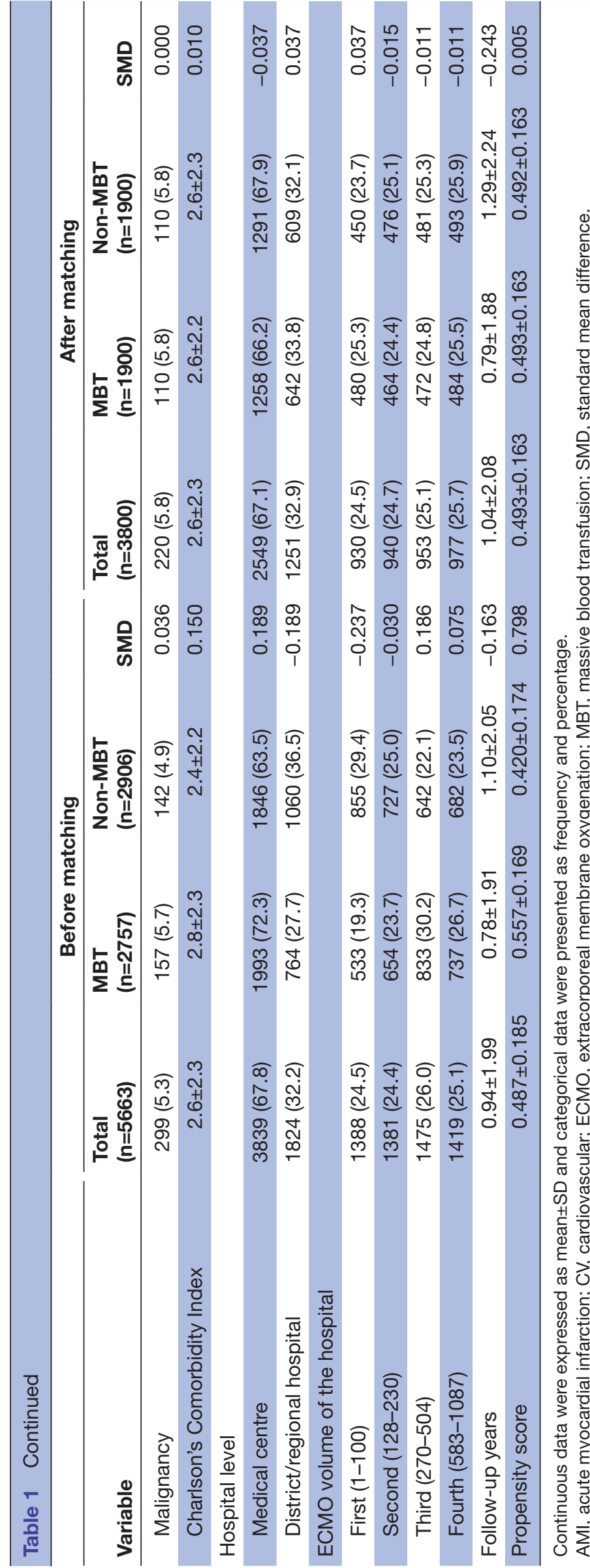




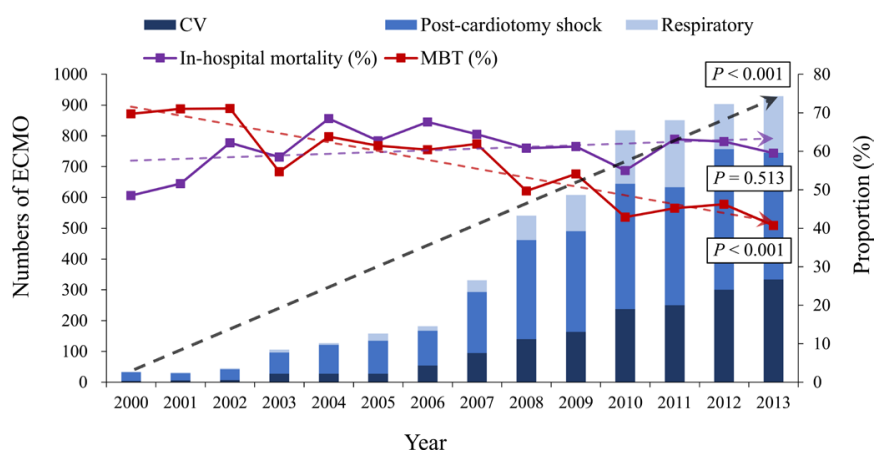

Figure 2 The epidemiology of ECMO application in Taiwan from 2000 to 2013. CV, cardiovascular; ECMO, extracorporeal membrane oxygenation; MBT, massive blood transfusion.

table 1). However, the MBT group had greater risks of ESRD during the follow-up. In patients with PCS indication, the MBT group had higher risks of overall mortality after discharge (HR 1.63; 95\% CI 1.32 to 2.01 ), CV death (HR $1.51 ; 95 \%$ CI 1.02 to 2.26 ) and respiratory failure (HR 1.60; $95 \%$ CI 1.17 to 2.20) than the non-MBT group. In addition, the risk of ESRD during the follow-up was not significantly different between MBT and non-MBT groups among PCS patients (HR 1.57; 95\% CI 0.80 to 3.07) (online supplementary table 2).
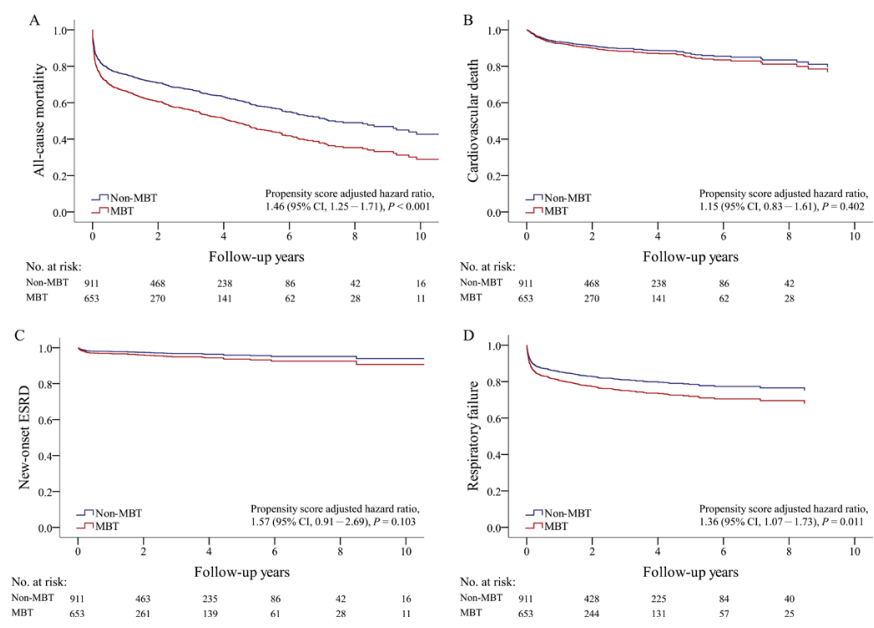

Figure 3 Propensity score adjusted (fitting) survival of (A) all-cause mortality, (B) cardiovascular death, (C) new-onset ESRD and (D) respiratory failure in the MBT and non-MBT groups in patients who survived the index ECMO admission. ECMO, extracorporeal oxygenation; ESRD, end-stage renal disease; MBT, massive blood transfusion.

\section{Effect of blood transfusion amount and transfusion ratio on in-hospital mortality}

Figure 4A illustrates the dose-dependent relationship between the transfused ratio (FFP/RBC) and in-hospital mortality risk. The cut-off points of the ratio were set as 0.5 and 1.0. Group 3 (ratio $>1.0$ ) had a higher in-hospital mortality than group 1 (ratio $<0.5 ; 75.2 \%$ vs $64.6 \%$; OR

Table 2 In-hospital complications and outcomes in the propensity score matched cohort

\begin{tabular}{|c|c|c|c|c|c|}
\hline \multirow[b]{2}{*}{ Outcome } & \multirow{2}{*}{$\begin{array}{l}\text { Total } \\
(\mathrm{n}=3800)\end{array}$} & \multirow{2}{*}{$\begin{array}{l}\text { MBT } \\
(n=1900)\end{array}$} & \multirow{2}{*}{$\begin{array}{l}\text { Non-MBT } \\
(n=1900)\end{array}$} & \multicolumn{2}{|c|}{ MBT versus non-MBT } \\
\hline & & & & B/OR (95\% Cl) & $P$ value \\
\hline \multicolumn{6}{|l|}{ Categorical parameter } \\
\hline In-hospital mortality & $2236(58.8)$ & $1247(65.6)$ & $989(52.1)$ & 1.74 (1.53 to 1.98$)$ & $<0.001$ \\
\hline New-onset stroke & $196(5.2)$ & $98(5.2)$ & $98(5.2)$ & $1.00(0.75$ to 1.34$)$ & 1.000 \\
\hline New-onset ischaemic stroke & $139(3.7)$ & $64(3.4)$ & $75(3.9)$ & 0.85 (0.61 to 1.19$)$ & 0.344 \\
\hline New-onset haemorrhagic stroke & $62(1.6)$ & $34(1.8)$ & $28(1.5)$ & 1.22 (0.74 to 2.03 ) & 0.439 \\
\hline Sepsis & $808(21.3)$ & $458(24.1)$ & $350(18.4)$ & 1.42 (1.21 to 1.67$)$ & $<0.001$ \\
\hline Fasciotomy or amputation & $44(1.2)$ & $31(1.6)$ & $13(0.7)$ & 2.39 (1.25 to 4.56$)$ & 0.009 \\
\hline Respiratory failure & $1843(48.5)$ & $976(51.4)$ & $867(45.6)$ & 1.27 (1.11 to 1.45$)$ & $<0.001$ \\
\hline Acute kidney injury & $1752(46.1)$ & 1108 (58.3) & 644 (33.9) & 2.73 (2.37 to 3.13$)$ & $<0.001$ \\
\hline \multicolumn{6}{|l|}{ Continuous parameter } \\
\hline PRBC amount & $13.2 \pm 13.7$ & $22.3 \pm 14.1$ & $4.1 \pm 3.1$ & 18.17 (17.52 to 18.82$)$ & $<0.001$ \\
\hline FFP amount & $9.5 \pm 15.8$ & $16.2 \pm 19.4$ & $2.8 \pm 5.5$ & 13.39 (12.49 to 14.29$)$ & $<0.001$ \\
\hline Platelet amount & $29.7 \pm 41.2$ & $50.4 \pm 47.8$ & $9.1 \pm 16.2$ & 41.31 (39.03 to 43.58$)$ & $<0.001$ \\
\hline ECMO support duration (days) & $3.6 \pm 3.7$ & $4.7 \pm 4.1$ & $2.5 \pm 2.9$ & 2.19 (1.97 to 2.41$)$ & $<0.001$ \\
\hline Ventilator (days) & $13.8 \pm 16.4$ & $16.8 \pm 17.1$ & $10.8 \pm 15.0$ & 5.95 (4.92 to 6.98$)$ & $<0.001$ \\
\hline ICU duration (days) & $15.4 \pm 16.7$ & $18.5 \pm 17.6$ & $12.3 \pm 15.3$ & 6.11 (5.06 to 7.16$)$ & $<0.001$ \\
\hline Hospital stays (days) & $22.6 \pm 18.9$ & $24.8 \pm 18.8$ & $20.5 \pm 18.8$ & 4.24 (3.06 to 5.42$)$ & $<0.001$ \\
\hline Inpatient medical expenditure (NTD $\left.\times 10^{4}\right)$ & $85.8 \pm 66.6$ & $103.1 \pm 66.9$ & $68.5 \pm 61.7$ & 34.61 (30.63 to 38.59$)$ & $<0.001$ \\
\hline
\end{tabular}

Continuous data were expressed as mean $\pm S D$ and categorical data were presented as frequency and percentage.

$\mathrm{B}$, regression coefficient; ECMO, extracorporeal membranes oxygenation; FFP, fresh frozen plasma; ICU, intensive care unit; MBT, massive blood transfusion; NTD, New Taiwan Dollar; PRBC, packed red blood cells. 
A

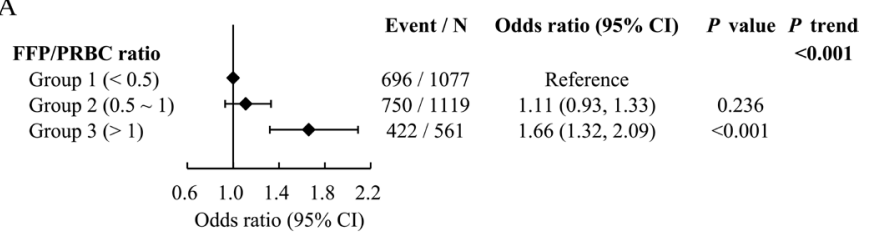

B

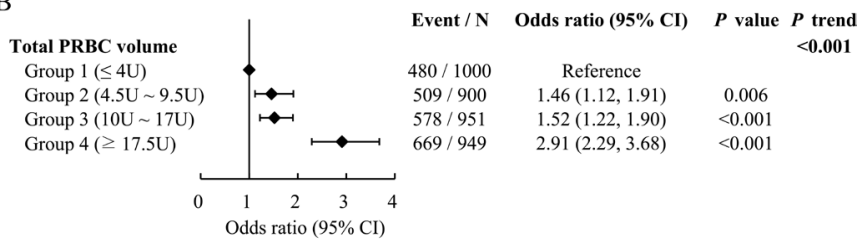

$\mathrm{C}$

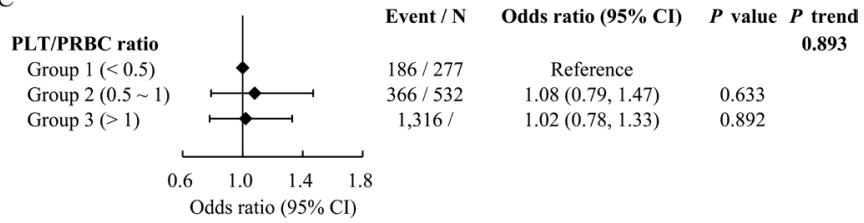

Figure 4 The dose-dependent relationship between $(A)$ the transfused ratio (FFP/PRBC), (B) total PRBC volume and (C) the transfused ration (PLT/PRBC) and risks of in-hospital mortality. FFP, fresh frozen plasma; PRBC, packed red blood cell; PLT, platelet.

1.66; $95 \%$ CI 1.32 to 2.09). Furthermore, the effect of the transfused amount of RBCs on in-hospital mortality was also examined in the subgroup analysis (figure 4B). However, the platelet to RBC transfusion ration had no correlation with in-hospital mortality risk (figure $4 \mathrm{C}$ ).

The actual transfused units of RBC were equally sorted into four categories, revealing that the greater the volume of transfused RBC, the higher the in-hospital mortality rate ( $\mathrm{p}$ for trend $<0.001$ ). For the third and fourth quartile groups, $60.8 \%$ and $70.5 \%$ of patients died during the index hospitalisation, respectively.

\section{DISCUSSION}

Using the Taiwan National Database, this study aimed to compare the long-term outcome of patients receiving MBT during ECMO support with that of patients who did not receive MBT. Patients with MBT had higher in-hospital mortality $(47.0 \%)$ and all-cause mortality (19.8\%) during the follow-up and had a higher risk of ECMO-related complications, such as sepsis, respiratory failure and acute kidney injury, than those without MBT. A higher cumulative incidence of respiratory failure was also found among MBT patients after index admission. Furthermore, the amount of transfused RBCs had a positive dose-dependent effect on in-hospital mortality.

Bleeding requiring blood transfusion is a common adverse complication of ECMO support. Intraoperative use of cardiopulmonary bypass with high heparinisation level, reduced platelet count, consumptive coagulopathy, haemolysis, therapeutic hypothermia and haemodilution might enhance the bleeding problem of patients with PCS under ECMO support. ${ }^{20}$ Rastan $e t$ al indicated that more than half of postcardiotomy patients with ECMO support even needed rethoracotomy because of refractory, excessive bleeding; these patients statistically had received perioperative $\mathrm{RBC}$ transfusion with up to 13 units during the first 48 hours. ${ }^{21}$

In the case of serious bleeding, RBC transfusion would be necessary and indicated. Bleeding is one of the leading causes of mortality for patients receiving ECMO.$^{22} \mathrm{RBC}$ transfusion might increase the incidence of ECMOrelated morbidity and in-hospital mortality. Lo Pinto et al concluded that RBC transfusion might be interrelated with higher ICU mortality and other severe complications, including acute renal failure, thromboembolic events and sepsis. ${ }^{23}$ According to several studies, in-hospital mortality is significantly higher in patients with larger amounts of RBC transfusion. ${ }^{24}{ }^{25}$ According to our results, in-hospital mortality was high for patients under any indication for ECMO support with massive RBC transfusion (figure 1B). Furthermore, patients with MBT had a significantly higher incidence of associated complications, such as sepsis, respiratory failure and acute kidney injury.

Acute lung injury due to MBT could be classified into transfusion-related acute injury (TRALI) and transfusionassociated circulatory overload. ${ }^{26}$ TRALI remains the major cause of transfusion-related mortality (37\%), and the incidence varies with plasma $(0.008 \%$ per unit $)$ and all blood products $\left(0.004 \%\right.$ per unit) ${ }^{27}$ In addition, ECMO itself may also be a deleterious factor of lung function for patients indicated for ECMO because of acute respiratory distress syndrome. ${ }^{28} 29$

The amount of transfused RBC influences the in-hospital mortality. ${ }^{30}$ Furthermore, Smith et al stated that each transfused RBC volume of $10 \mathrm{~mL} / \mathrm{kg} /$ day of ECMO would contribute to a $24 \%$ increase in the odds of in-hospital mortality. ${ }^{32}$ This finding is consistent with our study; the actual transfused RBC volume was positively associated with in-hospital mortality.

Meanwhile, MBT with a high FFP-to-PRBC ratio has benefits of lower mortality and better clinical outcomes in patients with trauma. ${ }^{33}$ However, another concept has been declared that a high FFP-to-PRBC ratio does not lead to a significant difference in 24 hours or 30-day mortality compared with a lower ratio $(1: 1$ vs $1: 2) .{ }^{34}$ For patients without trauma but with severe bleeding, a high transfusion FFP-to-PRBC ratio of greater than 1:2 might be appropriate for patients with trauma; however, this ratio seems not to have a positive impact on mortality rate in patients without trauma. ${ }^{35} 36$ In our study, a greater transfused amount of FFP was generally required in more serious coagulopathy situations with refractory bleeding and a worse prognosis. Therefore, MBT with a higher ratio (FFP-to-PRBC $\geq 1.0$ ) might result in a higher in-hospital mortality rate for patients under ECMO support. Considering the limited evidence, further research is needed to determine the ideal MBT ratio for patients under ECMO support.

Platelet dysfunction during ECMO support had been investigated with several possible mechanisms, such as 
enhanced platelet activation related to high shear stress, platelet adhesion/activation problem induced by an artificial extracorporeal circuit, and reduced binding of von Willebrand factor to platelet. ${ }^{6} 3738$ However, information about the suggested platelet transfusion strategy in clinical practice for ECMO patients is still limited. Only few investigations on paediatric patients under ECMO use indicated that a larger volume of platelet transfusion is associated with a higher risk for mortality and coagulopathy. ${ }^{39} 40$ Further studies for the outcomes of adult ECMO patients with platelet transfusion are required in the future.

In our study, patients with MBT during ECMO support had a higher cumulative incidence of respiratory failure after index hospitalisation during a follow-up. Blood transfusion and ECMO application could lead to pulmonary dysfunction in different ways. However, evidence on the relationship between MBT and respiratory failure after discharge remains unavailable. Thus, further research is necessary to investigate the possible mechanism of developing respiratory failure following index hospitalisation among patients with MBT.

Further analysis of the effect for different ECMO indications (PCS and non-PCS) on MBT proportion, in-hospital complication/mortality and cumulative incidences of adverse events after index hospitalisation are displayed in online supplementary tables 1 and 2. As for non-PCS population, no significant influence was observed on in-hospital mortality, cumulative incidence of all-cause mortality and respiratory failure after index hospitalisation, except for new-onset ESRD. In contrast, the PCS group had significant difference among in-hospital mortality, and cumulative incidence of negative events following index hospitalisation, excluding new-onset ESRD. Consequently, PCS patients not only were the main two-third resource of the MBT group but also dominantly affect both short-term and long-term outcomes.

\section{Limitation}

First, the features of the cohort study limited this study. Although this study was based on the NHIRD, which covers nearly the entire population of Taiwan, detailed patient information, such as vital signs, physical examination results, exact dosage of medication, laboratory examination data, clinical image reports and surgery/ procedure notes, were not recorded in the NHIRD. Therefore, we could not identify the severity of bleeding status, definite anticoagulation strategies, ECMO setting, bleeding site, exact timing of blood transfusion and the actual amount of transfused blood/kilogram/day. The important parameters of ECMO, such as cannula size, cannula insertion site (central or peripheral), speed of the centrifugal pump and support mode (veno-venous, veno-arterial or switch one from the other), are factors reportedly associated with various outcomes. The exact dose of anticoagulation drugs and the severity/site of bleeding may also affect the morbidity and mortality among these patients. The patient cohort included patients with different risk profile with respect to the bleeding complications. Nevertheless, we could hardly get access to the all risk profile for analysis from NHIRD.

Second, the NHI data after 2013 (data of 2018 are available soon in March or April 2020) are available only in the National Health Informatics Project, which is managed by Health and Welfare Data Science Center. Our available data source was NHIRD, which was maintained by the National Health Research Institutes, but it was no longer updated after 2013. Therefore, we could only acquire legal access to analyse data during the period between 2000 and 2013. However, the trend of number, proportion of indications and outcomes for ECMO use might change with time. Hence, further relevant research with updated data is needed.

Third, the annual growth of ECMO use is also an interesting point. However, under the reimbursement regulation, the ECMO application is liberal without consideration on medical cost. Furthermore, the validation and effectiveness of ECMO in Taiwan have been examined by numerous retrospective articles. ${ }^{1541}$

\section{CONCLUSION}

Patients with MBT during ECMO support had a worse outcome than the non-MBT population. Higher risks of in-hospital mortality and all-cause mortality were noted among the MBT patients. The incidences of post-ECMO complications, such as sepsis, respiratory failure and acute kidney injury, also were higher in the MBT group. Moreover, a positive dose-dependent relationship was noted between the amount of transfused RBC and in-hospital mortality.

\section{Author affiliations}

${ }^{1}$ Department of Anesthesiology, Chang Gung Memorial Hospital, Linkou Medical Center, Taoyuan, Taiwan

${ }^{2}$ Division of Thoracic and Cardiovascular Surgery, Department of Surgery, Chang Gung Memorial Hospital, Linkou Medical Center, Chang Gung University, Taoyuan City, Taiwan

${ }^{3}$ Department of Medicine, Chang Gung University, Linkou, Taipei, Taiwan, ROC ${ }^{4}$ Department of Cardiology, Chang Gung Memorial Hospital, Linkou Medical Center, Taoyuan City, Taiwan

${ }^{5}$ Department of Anesthesiology, Xiamen Changgung Hospital, Taoyuan, Taiwan

Acknowledgements The study was supported by a grant from the Chang Gung Medical Research Project (BMRPC19, CMPRG3D1471, CMRPG3D1472, CMRPG3D1473, CORPG3G0591, CMRPG3G0601, CORPG3G0611, CORPG3G0621) Chang Gung Memorial Hospital, Linkou, Taiwan, and the Ministry of Science and Technology, Taiwan (MOST 106-2314-B-182A-061-MY2). F-TC, I-L Su, S-WC, VC-CW, H-A Lee, P-CT and A-HC had full access to all the data used in the study and take responsibility for the integrity of the data and accuracy of analysis. The authors thank Alfred Hsing-Fed Lin and Zoe Ya-Jhu Syu for their assistance in statistical analysis.

Contributors F-TC, S-WC, VC-CW, K-CH, S-HC and P-CT make substantial contributions to conception and design, acquisition of data, analysis and interpretation of data. F-TC, S-WC and A-HC participate in drafting the article or revising it critically for important intellectual content. All authors give final approval of the version to be submitted and any revised version.

Funding The authors have not declared a specific grant for this research from any funding agency in the public, commercial or not-for-profit sectors.

Competing interests None declared. 
Patient and public involvement Patients and/or the public were not involved in the design, or conduct, or reporting, or dissemination plans of this research.

Patient consent for publication Not required.

Ethics approval Ethical approval was granted by the Institutional Review Board/ Ethics Committee of Chang Gung Memorial Hospital, Linkou Medical Center (approval no. 104-7990B).

Provenance and peer review Not commissioned; externally peer reviewed.

Data availability statement No data are available.

Open access This is an open access article distributed in accordance with the Creative Commons Attribution Non Commercial (CC BY-NC 4.0) license, which permits others to distribute, remix, adapt, build upon this work non-commercially, and license their derivative works on different terms, provided the original work is properly cited, appropriate credit is given, any changes made indicated, and the use is non-commercial. See: http://creativecommons.org/licenses/by-nc/4.0/.

\section{ORCID iDs}

Fang-Ting Chen http://orcid.org/0000-0002-9305-8396

An-Hsun Chou http://orcid.org/0000-0002-1711-5424

\section{REFERENCES}

1 Abrams D, Combes A, Brodie D. Extracorporeal membrane oxygenation in cardiopulmonary disease in adults. J Am Coll Cardiol 2014;63:2769-78.

2 Diddle JW, Almodovar MC, Rajagopal SK, et al. Extracorporeal membrane oxygenation for the support of adults with acute myocarditis. Crit Care Med 2015;43:1016-25.

3 Mosier JM, Kelsey M, Raz Y, et al. Extracorporeal membrane oxygenation (ECMO) for critically ill adults in the emergency department: history, current applications, and future directions. Crit Care 2015;19:431.

4 Kanji HD, Schulze CJ, Oreopoulos A, et al. Peripheral versus central cannulation for extracorporeal membrane oxygenation: a comparison of limb ischemia and transfusion requirements. Thorac Cardiovasc Surg 2010;58:459-62.

5 Schmidt M, Stewart C, Bailey M, et al. Mechanical ventilation management during extracorporeal membrane oxygenation for acute respiratory distress syndrome: a retrospective international multicenter study. Crit Care Med 2015;43:654-64.

6 Thomas J, Kostousov V, Teruya J. Bleeding and thrombotic complications in the use of extracorporeal membrane oxygenation. Semin Thromb Hemost 2018;44:020-9.

7 Sihler KC, Napolitano LM. Complications of massive transfusion. Chest 2010;137:209-20.

8 Loor G, Rajeswaran J, Li L, et al. The least of 3 evils: exposure to red blood cell transfusion, anemia, or both? J Thorac Cardiovasc Surg 2013;146:1480-7.

9 Toy P, Popovsky MA, Abraham E, et al. Transfusion-related acute lung injury: definition and review. Crit Care Med 2005;33:721-6.

10 Lee H-A, Cheng Y-T, Wu VC-C, et al. Nationwide cohort study of mitral valve repair versus replacement for infective endocarditis. J Thorac Cardiovasc Surg 2018;156:1473-83.

11 Chen C-C, Chen T-H, Tu P-H, et al. Long-term outcomes for patients with stroke after coronary and valve surgery. Ann Thorac Surg 2018;106:85-91.

12 Chen S-W, Wu VC-C, Lin Y-S, et al. Propensity score matched analysis of mechanical vs. bioprosthetic valve replacement in patients with previous stroke. Circ J 2018;82:2041-8.

13 Chou A-H, Chen C-C, Lin Y-S, et al. A population-based analysis of endovascular aortic stent graft therapy in patients with liver cirrhosis. J Vasc Surg 2019;69:1395-404.

14 Pham HP, Shaz BH. Update on massive transfusion. Br J Anaesth 2013;111:i71-82

15 Chen S-W, Tsai F-C, Lin Y-S, et al. Long-term outcomes of extracorporeal membrane oxygenation support for postcardiotomy shock. J Thorac Cardiovasc Surg 2017;154:469-77.

16 Cheng C-L, Chien $\mathrm{H}-\mathrm{C}$, Lee $\mathrm{C}-\mathrm{H}$, et al. Validity of in-hospital mortality data among patients with acute myocardial infarction or stroke in national health insurance research database in Taiwan. Int J Cardiol 2015;201:96-101.

17 Wu C-Y, Chen Y-J, Ho HJ, CY W, HJ H, et al. Association between nucleoside analogues and risk of hepatitis $B$ virus-related hepatocellular carcinoma recurrence following liver resection. JAMA 2012;308:1906-14.
18 Chen S-W, Chang C-H, Lin Y-S, et al. Effect of dialysis dependence and duration on post-coronary artery bypass grafting outcomes in patients with chronic kidney disease: a nationwide cohort study in Asia. Int J Cardiol 2016;223:65-71.

19 Elze MC, Gregson J, Baber U, et al. Comparison of propensity score methods and covariate adjustment: evaluation in 4 cardiovascular studies. J Am Coll Cardiol 2017;69:345-57.

20 Thiagarajan RR, Barbaro RP, Rycus PT, et al. Extracorporeal life support organization registry international report 2016. Asaio J 2017;63:60-7.

21 Rastan AJ, Dege A, Mohr M, et al. Early and late outcomes of 517 consecutive adult patients treated with extracorporeal membrane oxygenation for refractory postcardiotomy cardiogenic shock. $J$ Thorac Cardiovasc Surg 2010;139:302-11.

22 Murphy DA, Hockings LE, Andrews RK, et al. Extracorporeal membrane oxygenation-hemostatic complications. Transfus Med Rev 2015;29:90-101.

23 Lo Pinto H, Allyn J, Persichini R, et al. Predictors of red blood cell transfusion and its association with prognosis in patients undergoing extracorporeal membrane oxygenation. Int J Artif Organs 2018;41:644-52.

24 Swol J, Marschall C, Strauch JT, et al. Hematocrit and impact of transfusion in patients receiving extracorporeal life support. Perfusion 2018;33:546-52.

25 GuimbretièreG, AnselmiA, RoisneA, et al. Prognostic impact of blood product transfusion in VA and VV ECMO. Perfusion 2019;34:246-53.

26 Roubinian NH, Looney MR, Kor DJ, et al. Cytokines and clinical predictors in distinguishing pulmonary transfusion reactions. Transfusion 2015;55:1838-46.

27 Meyer DE, Reynolds JW, Hobbs R, et al. The incidence of transfusion-related acute lung injury at a large, urban tertiary medical center: a decade's experience. Anesth Analg 2018;127:444-9.

28 Lindén VB, Lidegran MK, Frisén G, et al. ECMO in ARDS: a long-term follow-up study regarding pulmonary morphology and function and health-related quality of life. Acta Anaesthesiol Scand 2009;53:489-95.

29 Hamutcu R, Nield TA, Garg M, et al. Long-term pulmonary sequelae in children who were treated with extracorporeal membrane oxygenation for neonatal respiratory failure. Pediatrics 2004;114:1292-6.

30 Mazzeffi M, Greenwood J, Tanaka K, et al. Bleeding, transfusion, and mortality on extracorporeal life support: ecls working group on thrombosis and hemostasis

31 Aubron C, Cheng AC, Pilcher D, et al. Factors associated with outcomes of patients on extracorporeal membrane oxygenation support: a 5-year cohort study. Crit Care 2013;17:R73.

32 Smith A, Hardison D, Bridges B, et al. Red blood cell transfusion volume and mortality among patients receiving extracorporeal membrane oxygenation. Perfusion 2013;28:54-60.

33 Stanworth SJ, Davenport R, Curry N, et al. Mortality from trauma haemorrhage and opportunities for improvement in transfusion practice. Br J Surg 2016;103:357-65.

34 Holcomb JB, Tilley BC, Baraniuk S, et al. Transfusion of plasma, platelets, and red blood cells in a 1:1:1 vs a 1:1:2 ratio and mortality in patients with severe trauma: the PROPPR randomized clinical trial. JAMA 2015;313:471-82.

35 McQuilten ZK, Crighton G, Brunskill S, et al. Optimal dose, timing and ratio of blood products in massive transfusion: results from a systematic review. Transfus Med Rev 2018;32:6-15.

36 Etchill EW, Myers SP, McDaniel LM, et al. Should all massively transfused patients be treated equally? an analysis of massive transfusion ratios in the Nontrauma setting. Crit Care Med 2017;45:1311-6.

37 Yoshimoto Y, Hasebe T, Takahashi K, et al. Ultrastructural characterization of surface-induced platelet activation on artificial materials by transmission electron microscopy. Microsc Res Tech 2013;76:342-9.

38 Chen Z, Mondal NK, Zheng S, et al. High shear induces platelet dysfunction leading to enhanced thrombotic propensity and diminished hemostatic capacity. Platelets 2019;30:112-9.

39 Saini A, West AN, Harrell C, et al. Platelet transfusions in the PICU: does disease severity matter? Pediatr Crit Care Med 2018;19:e472-8.

40 Cashen K, Dalton H, Reeder RW, et al. Platelet transfusion practice and related outcomes in pediatric extracorporeal membrane oxygenation. Pediatr Crit Care Med 2020;21:178-85.

41 Wu M-Y, Lin P-J, Lee M-Y, et al. Using extracorporeal life support to resuscitate adult postcardiotomy cardiogenic shock: treatment strategies and predictors of short-term and midterm survival. Resuscitation 2010;81:1111-6. 